\title{
Francisella RNA polymerase contains a heterodimer of non-identical $\alpha$ subunits
}

\author{
Damir Mukhamedyarov ${ }^{1}$, Kira S Makarova ${ }^{2}$, Konstantin Severinov ${ }^{1,3}$ and Konstantin Kuznedelov ${ }^{1 *}$
}

\begin{abstract}
Background: All sequenced genomes of representatives of the Francisella genus contain two rpoA genes, which encode non-identical RNA polymerase (RNAP) subunits, $\alpha 1$ and $\alpha 2$. In all other bacteria studied to date, a dimer of identical $\alpha$ subunits initiates the assembly of the catalytically proficient RNAP core (subunit composition $\alpha_{2} \beta \beta^{\prime}$ ). Based on an observation that both $\alpha 1$ and $\alpha 2$ are incorporated into Francisella RNAP, Charity et al. (2007) previously suggested that up to four different species of RNAP core enzyme might form in the same Francisella cell.

Results: By in vitro assembly from fully denatured state, we determined that both Francisella $\alpha$ subunits are required for efficient dimerization; no homodimer formation was detected. Bacterial two-hybrid system analysis likewise indicated strong interactions between the $\alpha 1$ and $\alpha 2 \mathrm{~N}$-terminal domains (NTDs, responsible for dimerization). NTDs of $\alpha 2$ did not interact detectably, while weak interaction between $\alpha 1$ NTDs was observed. This weak homotypic interaction may explain low-level transcription activity observed in in vitro RNAP reconstitution reactions containing Francisella large subunits $\left(\beta^{\prime}, \beta\right)$ and $\alpha 1$. No activity was observed with RNAP reconstitution reactions containing $\alpha 2$, while robust transcription activity was detected in reactions containing $\alpha 1$ and $\alpha 2$. Phylogenetic analysis based on RpoA resulted in a tree compatible with standard bacterial taxonomy with both Francisella RpoA branches positioned within $\gamma$-proteobacteria. The observed phylogeny and analysis of constrained trees are compatible with Francisella lineage-specific rpoA duplication followed by acceleration of evolutionary rate and subfunctionalization.
\end{abstract}

Conclusions: The results strongly suggest that most Francisella RNAP contains $\alpha$ heterodimer with a minor subfraction possibly containing $\alpha 1$ homodimer. Comparative sequence analysis suggests that this heterodimer is oriented, in a sense that only one monomer, $\alpha 1$, interacts with the $\beta$ subunit during the $\alpha_{2} \beta$ RNAP subassembly formation. Most likely the two rpoA copies in Francisella have emerged through a lineage-specific duplication followed by subfunctionalization of interacting paralogs.

\section{Background}

Bioinformatics analysis reveals that two paralogous rpoA genes, each encoding non-identical proteins homologous to bacterial RNA polymerase (RNAP) $\alpha$ subunits, are present in the genome of Francisella tularensis [1]. The bacterial RNAP core enzyme has subunit composition $\alpha_{2} \beta \beta^{\prime}$. Variations including fusion of the largest subunits, $\beta$ and $\beta$ ', in Helicobacter and Wolinella genera $[2,3]$, and split the largest subunit in some cyanobacteria

\footnotetext{
* Correspondence: kuznedelo@waksman.rutgers.edu

'Department of Biochemistry and Molecular Biology and Waksman Institute of Microbiology, Rutgers, the State University of New Jersey, Piscataway, NJ 08854, USA

Full list of author information is available at the end of the article
}

[4] have been reported, but overall, the subunit composition of RNAP core is conserved. The $\alpha$ subunit homodimer initiates bacterial RNAP assembly. The $\alpha$ subunit monomers dimerize through their $\mathrm{N}$-terminal domain (NTD) [5,6]. The C-terminal domain (CTD) is connected to NTD through a flexible tether [7]. The $\alpha$ CTD is not required for assembly but is involved in transcriptional regulation [8-10]. The $\alpha$ NTD homodimer provides a platform for interaction with the two large RNAP subunits [11,12]. Determinants in $\alpha$ important for interactions with $\beta$ and $\beta^{\prime}$ subunits have been localized by mutagenesis and hydroxyl-radical footprinting studies [5-8,13-15]. Substitutions at positions 45 and 48 of Escherichia coli $\alpha$ subunit completely (R45A) or
Ciomed Central

(ㄷ) 2011 Mukhamedyarov et al; licensee BioMed Central Ltd. This is an Open Access article distributed under the terms of the Creative Commons Attribution License (http://creativecommons.org/licenses/by/2.0), which permits unrestricted use, distribution, and reproduction in any medium, provided the original work is properly cited. 
partially (L48A) prevented formation of the $\alpha_{2} \beta$ RNAP subassembly [16]. Two point substitutions at positions 86 and 173, and two-amino-acid insertions at positions 180 and 200 of $E$. coli $\alpha$ caused defects in $\beta^{\prime}$ binding without affecting the $\alpha_{2} \beta$ assembly formation $[16,17]$. RNAP containing oriented $E$. coli $\alpha$ heterodimers have been prepared both in vitro, by reconstitution from recombinant subunits, and in vivo, by co-expression of genes for recombinant subunits, by using one $\alpha$ subunit lacking the R45A substitution and one $\alpha$ subunit having the R45A substitution $[18,19]$. Functional analysis of RNAP containing oriented $\alpha$ heterodimers confirmed that asymmetrical arrangement of $\alpha$ leads to non-identical functions of each monomer in transcription regulation $[18,19]$.

RNAP core enzymes from archaea and eukaryotes contain homologs of each of the bacterial RNAP core subunits. However, rather than having two identical $\alpha$ subunit homologs, they contain two different $\alpha$-like polypeptides (RPB3 and RPB11 in the case of eukaryotic RNAP II) that form a heterodimer, which serves as a platform for RNAP assembly [20].

The presence of two different genes (rpoA1 and rpoA2) in the genome of Francisella suggests that up to four RNAP core enzymes differing in subunit composition could be present in the cells: two enzymes containing $\alpha$ homodimers, $(\alpha 1)_{2} \beta \beta^{\prime}$ and $(\alpha 2)_{2} \beta \beta^{\prime}$, and two enzymes containing $\alpha$ heterodimers, $(\alpha 1 \alpha 2) \beta \beta^{\prime}$ and $(\alpha 2 \alpha 1) \beta \beta^{\prime}[1]$. The heterodimers could differ from one another with respect to which $\alpha$ interacts with the $\beta$ subunit of RNAP and which $\alpha$ interacts with $\beta^{\prime}[18,19]$. Promoter recognition properties of RNAP holoenzymes formed from these different core enzyme molecules may differ, since CTD of $\alpha 1$ and $\alpha 2$ may be capable of different protein-DNA and protein-protein interactions during transcription initiation [18,19]. Further, if holoenzymes containing RNAP core enzymes of different composition indeed respond differently to transcription factors and elements, then $F$. tularensis may regulate the spectrum of expressed genes by altering the relative ratio of core enzymes with different $\alpha$ subunit composition, which would be a novel paradigm of transcription regulation in bacteria.

Evidence of that both $\alpha 1$ and $\alpha 2$ subunits are incorporated into $F$. tularensis RNAP has been reported earlier by Charity et al. [1]. These authors demonstrated that RNAP affinity purified from $F$. tularensis strain expressing the $\beta$ ' subunit with fused TAP-tag contained both $\alpha 1$ and $\alpha 2$. These experiments clearly show that both rpoA genes are active and their products are components of RNAP but do not inform about the actual subunit composition of $F$. tularensis RNAP. Based on predicted dimerization determinants in other bacteria [21], Charity et al. hypothesized that $\alpha 1$ and $\alpha 2$ might exclusively form either homodimers or heterodimers [1]. In the present study, we describe the results of in vitro analysis of assembly of RNAP from F. tularensis subspecies novicida. Our results indicate that RNAP core containing an $\alpha$ heterodimer is the main, perhaps the only, species of RNAP in this organism. We further present results of phylogenetic analysis that provide a plausible scenario for the appearance of two paralogous rpoA genes in the Francisella lineage.

\section{Results}

\section{F. tularensis $\alpha$ heterodimer but not homodimers efficiently assembles in vitro}

To experimentally address the ability of F. tularensis RNAP $\alpha$ subunits to form homo- and heterodimers, we investigated the ability of recombinant $F$. tularensis $\alpha$ subunit proteins with $\mathrm{C}$-terminal $\mathrm{His}_{6}$-tags to pull down untagged counterparts during ion metal affinity chromatography. As shown previously, F. tularensis RNAP $\alpha$ subunits have different electrophoretic mobilities, with $\alpha 1$ migrating significantly faster than $\alpha 2$ [1]. In addition, $\mathrm{His}_{6}$-tags alter electrophoretic mobility of both $\alpha 1$ and $\alpha 2$ enough to separate tagged and untagged $\alpha$ subunits of the same kind (Figure 1).

Therefore, because each of the four proteins used in the pull-down assay has a characteristic electrophoretic mobility, it is possible to detect the efficiency of both hetero- and homodimer formation. Various pairwise combinations of $\alpha$ subunits were mixed at denaturing conditions ( $6 \mathrm{M}$ guanidinium chloride), the denaturing reagent was removed by dialysis at conditions favouring bacterial RNAP assembly from isolated subunits [22], and reconstitution reactions (labelled "L" on Figure 1) were loaded on $\mathrm{Ni}^{2+}$-affinity columns. Flow-through (F) was collected and retained protein was eluted (E) with different concentrations of imidazole in the buffer. Aliquots of each fraction were next analyzed by SDS-PAGE. As can be seen from Figure 1 (top panel), no co-immobilization of untagged $\alpha$ subunit in reactions that contained tagged and untagged versions of subunit of the same kind was detected. In contrast, heterodimers were readily detected when either $\alpha 1 \mathrm{His}_{6}$ or $\alpha 2 \mathrm{His}_{6}$ were used as "baits" for co-immobilization of, respectively, $\alpha 2$ or $\alpha 1$ (Figure 1, bottom panel). We conclude that F. tularensis RNAP $\alpha$ subunits do not appreciably form homodimers, at least at the conditions of in vitro RNAP assembly.

\section{$\alpha 1$ NTD and $\alpha 2$ NTD efficiently interact in bacterial 2- hybrid system}

RNAP $\alpha$ subunit is a two-domain protein, with its $\mathrm{N}$ terminal domain being primarily responsible for dimerization and interaction with large RNAP subunits, while the C-terminal domain, CTD, which is connected to NTD through a flexible linker, is primarily responsible for 


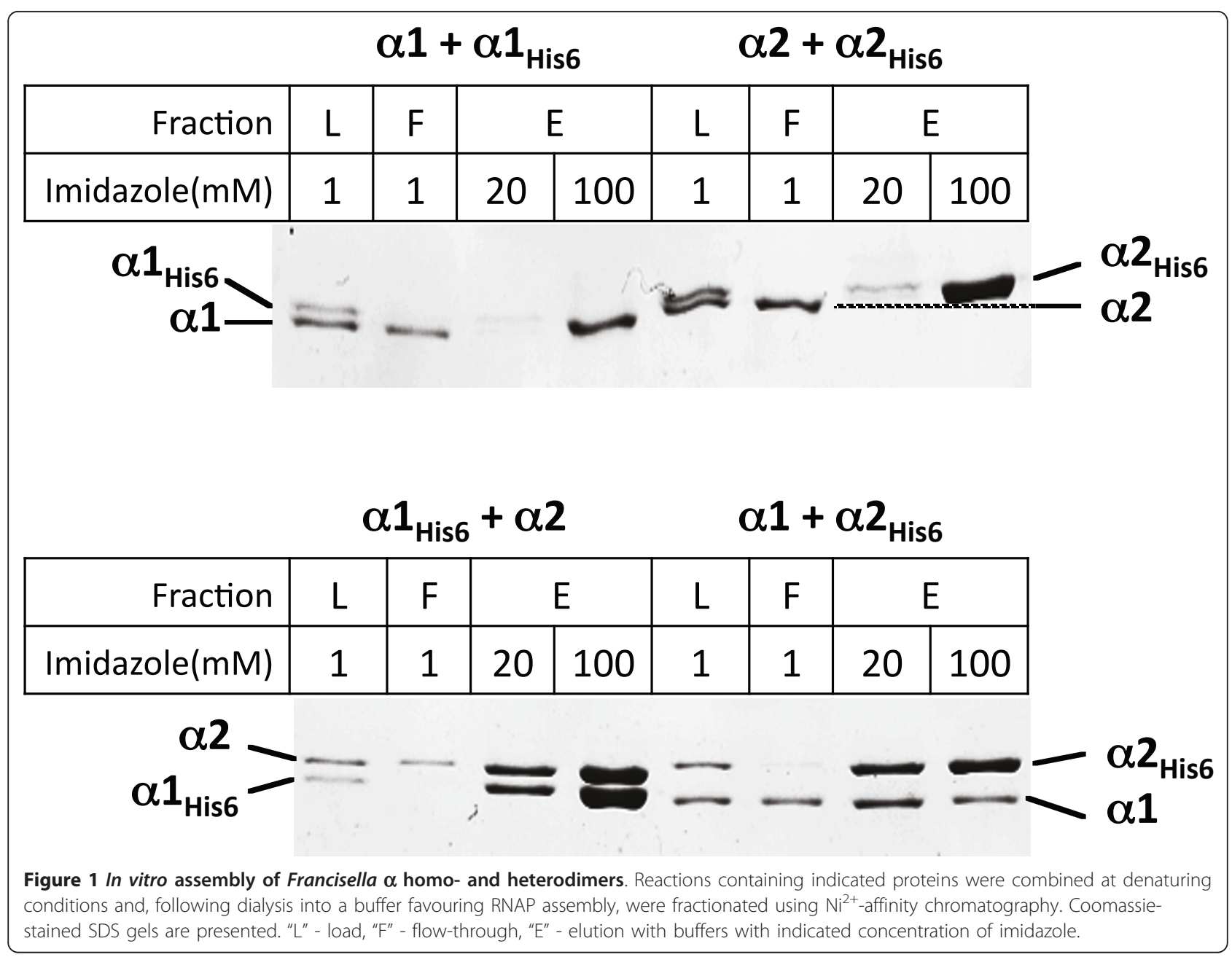

interactions with transcription factors and DNA upstream of the -35 promoter element [23]. Weak dimerization of isolated $\alpha$ CTD has been reported and may be of regulatory significance [7]. To independently study dimerization of various domains of $F$. tularensis $\alpha$ subunits, we used the bacterial two-hybrid system [24]. Eight two-hybrid plasmids expressing bait and prey fusions of each $\alpha$ domain were constructed and 16 pairwise combinations were tested. The results are presented in Table 1. As can be seen, in agreement with in vitro co-immobilization data, strong interactions between $\alpha$ NTDs of different kinds were detected. $\alpha$ CTDs did not appreciably interact with each other or with $\alpha$ NTDs. The level of homotypic interaction between $\alpha 1$ NTD was above the background, potentially indicating formation of $\alpha 1$ NTD homodimer, while the level of $\alpha 2$ homodimer formation was at the background level.

Formation of the $\alpha_{2} \beta$ subassembly in vitro

RNAP assembly follows a conserved pathway, whereby the $\beta$ subunit interacts with the $\alpha$ dimer, leading to the formation of $\alpha_{2} \beta$ - a stable intermediate of RNAP assembly that can be observed both in vivo and in vitro $[11,16]$. We performed in vitro RNAP assembly using His $_{6}$-tagged F. tularensis $\alpha$ subunits and untagged

Table 1 Bacterial two-hybrid analysis of interactions between domains of $\alpha 1$ and $\alpha 2$ subunits of $F$. tularensis RNAP

\begin{tabular}{ccccc}
\hline pBR $\alpha$ Ln+pAC $\lambda \mathrm{cl}$ & $\alpha 1$ NTD & $\alpha 1 C T D$ & $\alpha 2 N T D$ & $\alpha 2$ CTD \\
\hline$\alpha 1$ NTD & $171 \pm 28$ & $44 \pm 1$ & $\mathbf{1 4 1 2} \pm \mathbf{3 2 3}$ & $53 \pm 9$ \\
\hline$\alpha 1 C T D$ & $110 \pm 13$ & $95 \pm 8$ & $133 \pm 5$ & $99 \pm 16$ \\
\hline$\alpha 2$ NTD & $662 \pm 26$ & $47 \pm 9$ & $74 \pm 4$ & $49 \pm 3$ \\
\hline$\alpha 2$ CTD & $102 \pm 3$ & $101 \pm 1$ & $112 \pm 6$ & $86 \pm 9$ \\
\hline
\end{tabular}

Eight two-hybrid plasmids expressing bait ( $1^{\text {st }}$ column) and prey ( $1^{\text {st }}$ row) fusions of each $\alpha$ subunit domain were constructed and 16 pairwise combinations were tested in a reporter strain by measuring $\beta$-galactosidase activity (in Miller units). Each combination was tested at least three times independently. Mean and standard deviation values are presented. Three kinds of measurements were taken to determine background levels of $\beta$ galactosidase activity, which was found to be (in Miller units) $111 \pm 26$ in host reporter cells with no plasmids, $110 \pm 9$ in cells transformed with pBRaLN$\alpha 1$ NTD only, and $50 \pm 7$ in cells transformed with pAC $\lambda$ cl- $\alpha 1$ NTD only. 
recombinant $F$. tularensis $\beta$. The results indicated that $\beta$ was most efficiently immobilized when both $\alpha$ subunits were present in the assembly reaction (Figure 2A, lane 12). Only trace amounts of $\beta$ were co-immobilized in reactions containing $\alpha 2$ (Figure $2 \mathrm{~A}$, lane 8 ) and thus likely represented non-specific binding (note that an excess of $\alpha 2$ was used in this reaction). The amount of $\beta$ co-immobilized in reactions containing $\alpha 1$ (lane 4 ) was higher than the background but clearly less than that observed in reactions containing both $\alpha$ subunits. We conclude from these experiments that $\beta$ interacts most efficiently with $\alpha$ heterodimer. Detected interaction between $\beta$ and $\alpha 1$ can proceed through $\alpha 1$ monomer or, alternatively, the $\beta$ subunit may stimulate formation of the $\alpha 1$ homodimer.

\section{In vitro transcription by recombinant $F$. tularensis RNAP}

To validate data obtained using two-hybrid analysis and $\alpha$ dimer $/ \alpha_{2} \beta$ RNAP subassembly in vitro reconstitution, in vitro RNAP assembly and transcription experiments were performed. Three in vitro RNAP assembly reactions contained recombinant $F$. tularensis $\beta$ and $\beta$ ' subunits and $\alpha 1, \alpha 2$, or both $\alpha 1$ and $\alpha 2$ (the $\omega$ subunit was omitted from assembly reactions as it is not essential for

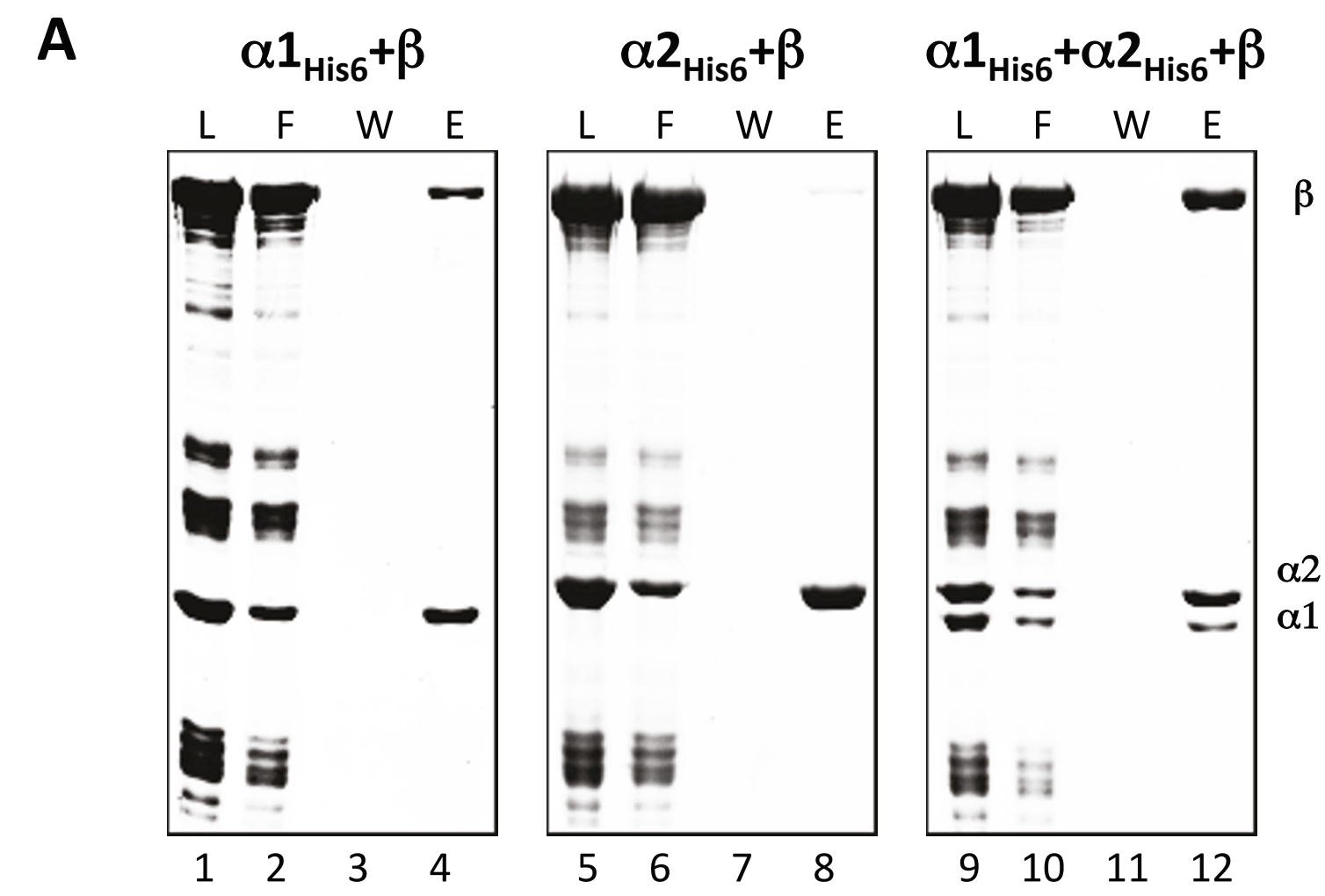

B

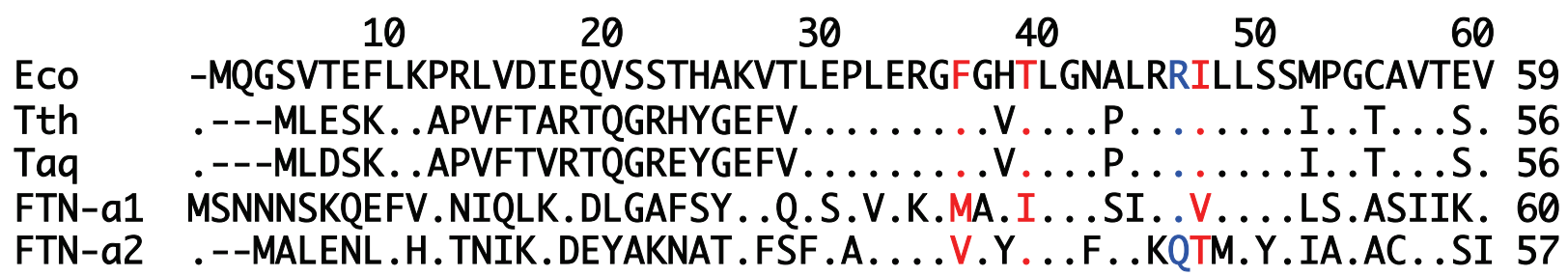

Figure 2 In vitro assembly of Francisella $\boldsymbol{\alpha}_{2} \boldsymbol{\beta}$ RNAP subassembly. A. Reactions were assembled and analyzed as described in Figure 1 legend. "W" -wash with excess of loading buffer. Proteins were eluted with a buffer containing $100 \mathrm{mM}$ imidazole. B. Sequence alignment of the $\alpha$ subunit segment involved in dimerization and interaction with the $\beta$ subunit. A segment of E. coli $\alpha$ subunit (amino acids 1-59) is shown at the top (single-letter amino acid code). Corresponding sequences from Thermus aquaticus (Taq), Thermus thermophilus (Tth), and Francisella tularensis RpoA variants are aligned below. Dots indicate identities, hyphens - gaps. Amino acids highlighted in red form a cluster important for $\alpha$ homodimer formation in E. coli. Amino acid highlighted in blue is responsible for the interaction with $\beta$. 
RNAP basic function $[25,26])$. Assembled RNAP reactions were passed through a gel-filtration column, fractions that eluted at retention times expected for RNAP core elution were collected and tested for transcription activity on a nucleic acid scaffold shown in Figure 3A. Nucleic acid scaffolds mimic the conformation of nucleic acids in transcription elongation complexes. RNAP complexes with nucleic acids scaffolds are catalytically active and serve as a convenient tool to study transcription elongation properties of the enzyme [27]. Reactions were combined with NTP, and elongation of radioactively labelled 8-nt RNA component of the scaffold ("RNA ${ }_{8}$ ") followed. The results are presented in Figure $3 \mathrm{~B}$. As can be seen, most efficient elongation of the RNA primer was observed in fractions obtained from RNAP assembly reaction containing both $\alpha$ subunits. Fractions of RNAP assembly reaction that contained $\alpha 2$ only were completely inactive. Fractions of RNAP assembly reaction containing $\alpha 1$ only demonstrated low but detectable transcription activity. We therefore conclude that $F$. tularensis RNAP assembles efficiently when both kinds of $\alpha$ subunits are present; $\alpha 2$ alone is unable to promote RNAP assembly; $\alpha 1$ alone supports

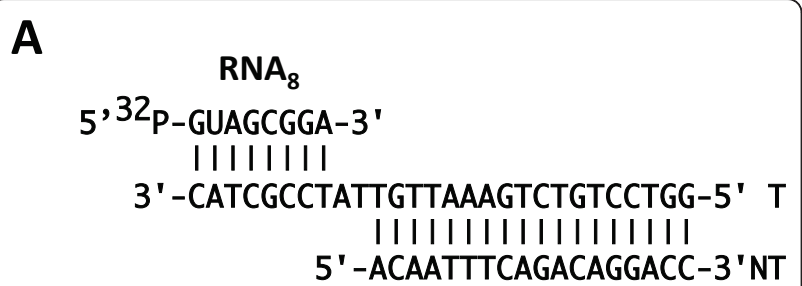

B

\begin{tabular}{|c|ccc|ccc|ccc|}
\hline Assembly & \multicolumn{2}{|c|}{$\alpha 1 \alpha 2 \beta \beta^{\prime}$} & \multicolumn{2}{|c|}{$\alpha 1 \beta \beta^{\prime}$} & \multicolumn{3}{|c|}{$\alpha 2 \beta \beta^{\prime}$} \\
\hline Fraction \# & 4 & 5 & 6 & 4 & 5 & 6 & 4 & 5 & 6 \\
\hline
\end{tabular}

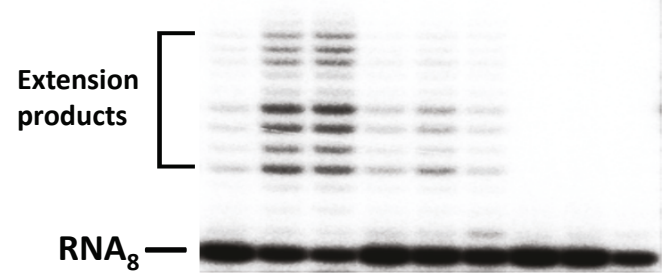

Figure 3 Transcription activity of in vitro assembled Francisella RNAP core enzymes. A. The structure of nucleic acid scaffold used to test assembled enzymes activities is schematically shown. B. Superose 6 fractions obtained after separation of in vitro RNAP assembly reactions containing indicated subunits were combined with nucleic acid scaffold containing a radioactively labelled RNA primer and reactions were supplemented with NTP. Reaction products were analyzed by denaturing PAGE. An autoradiograph is presented.
RNAP assembly, albeit with low efficiency, possibly due to low level of $\alpha 1$ homodimer formation.

\section{Evolution of RpoA in Francisella}

To gain insight into the evolution of two paralogs of RpoA in Francisella, we retrieved all RpoA sequences in all of the 1055 completely sequenced bacterial genomes available in the RefSeq database. We found that in addition to Francisella, two rpoA genes (rpoA1 and rpoA2) are present in several other genomes, namely in three Chloroflexus species (C. aggregans DSM 9485; C. aurantiacus J-10-fl; C. sp. Y-400-fl), in Streptomyces avermitilis MA-4680, in Psychromonas ingrahamii 37, and in Leptospira borgpetersenii serovar Hardjo bovis L550 (see also Additional file 1). In the latter two cases the two rpo $A$ copies are identical and are apparently the result of very recent genome segment duplications that also include a number of other genes.

In order to address an alternative possibility, that one of the RpoA paralogs in Francisella could have been horizontally transferred from a distant bacterial (other than $\gamma$-proteobacteria to which the Francisella genus belongs) lineage instead of arising through gene duplication, we reconstructed RpoA phylogenetic tree for a representative set of bacteria including those that contain rpo $A$ duplications listed above (Figure 4A). The resulting tree is generally very well compatible with bacterial taxonomy, which is not surprising considering the fact that RNAP subunits are among the best phylogenetic markers [28-30]. The position of both RpoA branches corresponding to Francisella within $\gamma$-proteobacteria is confidently supported by bootstrap analysis (bootstrap probability of 0.93 ). Thus, it is unlikely that any of the Francisella rpoA genes were transferred from outside of the $\gamma$-proteobacterial lineage. Branches leading to both Francisella RpoA proteins are extremely long, which might cause an artefact of the long-branch attraction, making the Francisella RpoA positioning unreliable. To test hypotheses for an alternative position of Francisella RpoA branches, we used RAxML [31] program to reconstruct a phylogenetic tree for $\gamma$-proteobacteria with $\beta$-proteobacteria outgroup (Figure 4B), made constrained trees and compared the maximum likelihood values for the best tree (Figure 4B) and constrained trees. The first constrained tree was designed to test a hypothesis of monophyly of two RpoA paralogs of Francisella; the second tree was designed to test a hypothesis of monophyly of both Francisella RpoA and of homologs from Coxiella, Legionella, and Thiomicrospira - species that are the closest taxonomic relatives of Francisella (Additional file 2). The analysis showed that none of the hypotheses could be rejected, suggesting that the positioning of RpoA at the root of $\gamma$-proteobacteria could be explained by long-branch attraction 


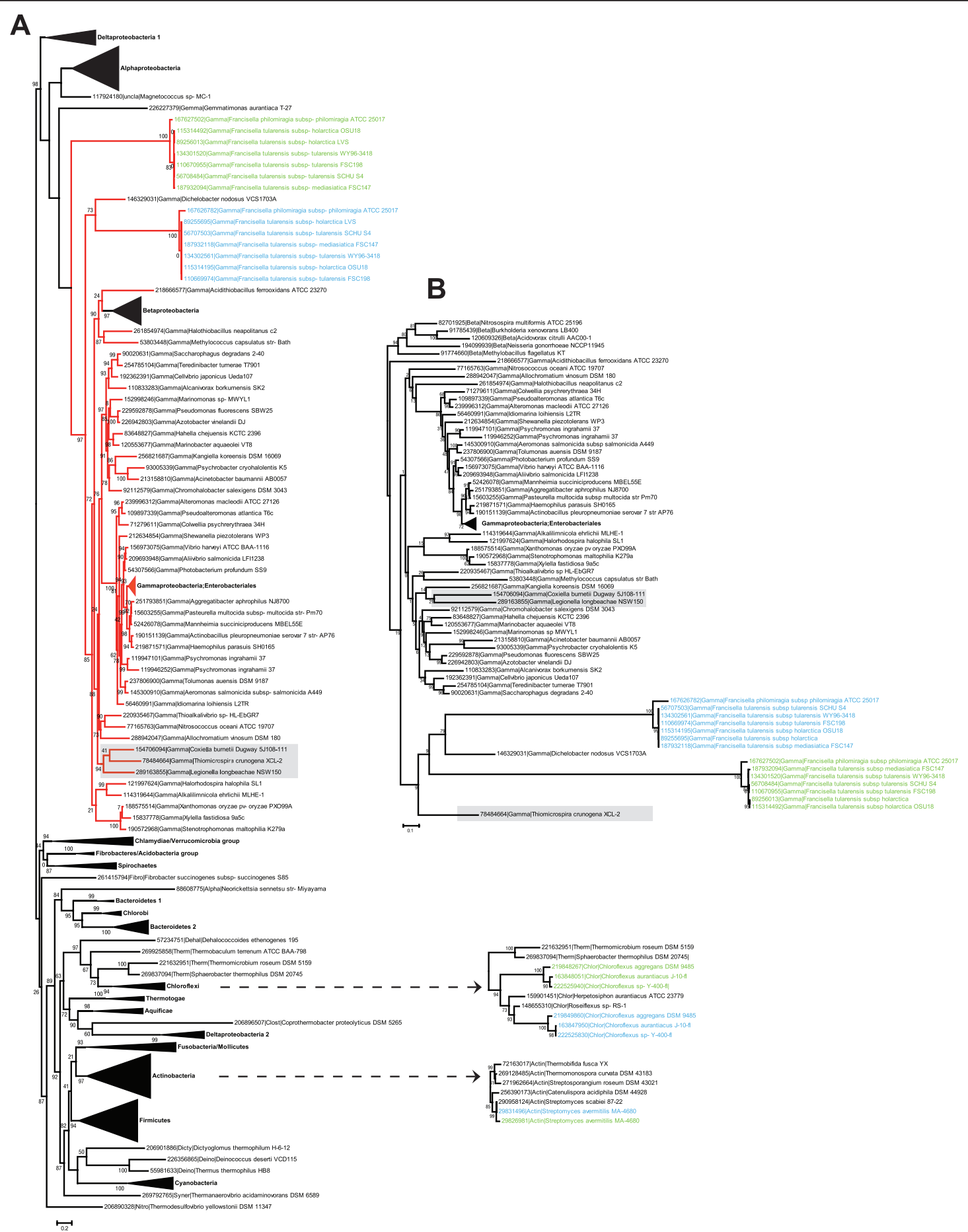

Figure 4 Phylogenetic trees of the bacterial RpoA. A. A set of 368 RpoA sequences from 355 representative genomes was aligned using MUSCLE program. The Maximum likelihood tree with bootstrap values applied was built for 246 informative aligned positions using FastTree program. B. A set of 84 RpoA sequences from 76 representative genomes of $\gamma$ - and $\beta$-proteobacteria was aligned using MUSCLE program. The Maximum likelihood tree with bootstrap values applied was built for 296 aligned informative positions using RAxML program. The evolutionary model for tree reconstruction (WAG with gamma-distributed evolutionary rates) has been selected using ProtTest program. Colour code: light blue - RpoA1 subunits encoded in ribosomal operons, the default location for all bacteria; light green - RpoA2 subunits encoded elsewhere in the genome. Dashed arrows point to subtrees with two other (non-Francisella) instances of RpoA duplication: Chloroflexus species and Streptomyces avermitilis. Each terminal tree node is labelled with GenBank Identifier (Gl) number, five-letter taxonomy code and full systematic name of an organism. The taxonomy code is the following: Gamma - $\gamma$-proteobacteria; Beta - $\beta$-proteobacteria; Alpha - $\alpha$ - proteobacteria; Clost Clostridia; Dicty - Dictyoglomi; Deino -Deinococcus/Thermus group; Syner - Synergistetes; Nitro - Nitrospirae; Therm -Thermomicrobia; Dehal Dehalococcoidetes; Fibro - Fibrobacteres/Acidobacteria group; Gemma - Gemmatimonadetes; uncla - unclassified. Compressed branches (black triangles) are labelled according to taxonomy. Branches leading to $\gamma$-proteobacteria are red. The shaded clade indicates close relatives of Fransicella according to taxonomy based on 16S rRNA and concatenated ribosomal proteins phylogeny. 
artefacts. Thus, we conclude that the two RpoA copies in Francisella most likely emerged through a duplication followed by acceleration of the evolutionary rates of both paralogs.

\section{Discussion}

Representatives of the bacterial genus Francisella are unusual with respect to RNAP composition, in that they contain two different $\alpha$ subunits encoded by two paralogous genes rpoA1 and rpoA2. The presence of two different $\alpha$ subunits in affinity-purified RNAP preparations [1] suggested that as many as four different species of RNAP core enzyme could be present in the single cell. Here, we studied Franicella RNAP by means of in vitro assembly. Our results show that Franicella $\alpha$ heterodimer $(\alpha 1 \alpha 2)$ efficiently assembles in vitro from fully denatured state and homodimers are not detected. Bacterial two-hybrid analysis indicates that in addition to efficient $\alpha$ heterodimer assembly, some dimerization of $\alpha 1$ may also occur. Thus, the efficiency of $\alpha$ dimerization is clearly a major factor that should affect subunit composition of Francisella RNAP. As was determined from crystal structure analysis, the main structural elements of the $\alpha$ dimer interface of $E$. coli are two $\alpha$-helices, $\mathrm{H} 1$ and $\mathrm{H} 3$, orthogonally oriented to each other [21]. These helices from one monomer participate in a coiled-coil-like interaction with their counterparts in the other monomer. Within these helices, Kannan et al. [32] identified a cluster of amino acids stabilizing interactions at the E. coli $\alpha$ dimer interface, with residues 35F, 38T, and $39 \mathrm{~L}$ emanating from one $\alpha$ monomer and residues 46I, 50S, and 227Q from another. Of particular interest are three of them, namely, 35F, 38T, and 46I, point mutations at which partially ( $\alpha$-T38A) [16] or completely $(\alpha-F 35 A, \alpha-I 46 S)$ [32] prevented the dimer formation. As can be seen from Figure 2B, amino acids at these positions are conserved (identical) between E. coli and Thermus, while amino acids in many of the corresponding positions in both $\alpha 1$ and $\alpha 2$ subunits from Francisella differ. Since these positions are critical for the dimer formation, it is reasonable to assume that some amino acids at these positions of Francisella $\alpha$ subunits, for example, $\alpha 1-36 \mathrm{M}, \alpha 1-39 \mathrm{I}, \alpha 2-33 \mathrm{~V}$, and $\alpha 2-47 \mathrm{~T}$, may be unfavourable to the assembly of homodimers. However, in the absence of crystal structure or systematic mutagenesis data, it is currently not possible to identify structural reasons for hetero- and homodimerization of Francisella $\alpha$ subunits.

During RNAP assembly in organisms where $\alpha$ subunit forms a homodimer, the $\beta$ subunit is free to interact with either $\alpha$ monomer to form the $\alpha_{2} \beta$ subassembly. The situation must be different in the case of $F$. tularensis, where $\alpha$ heterodimers form preferentially. In E. coli, an evolutionary conserved $\alpha$ subunit residue $\operatorname{Arg}^{45}$ is critical for $\beta$ subunit interaction with the $\alpha$ dimer $[16,18,19]$. In $F$. tularensis, the corresponding position in $\alpha 1$ also contains an arginine $\left(\mathrm{Arg}^{46}\right)$, while in $\alpha 2$ this position is occupied by glutamine $\left(\mathrm{Gln}^{42}\right.$; Figure $\left.2 \mathrm{~B}\right)$. Thus, it appears that $\beta$ subunit in $F$. tularensis will be specifically interacting with the $\alpha$ heterodimer through $\alpha 1$. That $\alpha 1$ contains determinants for interactions with $\beta$ also follows from results of the $\alpha_{2} \beta$ subassembly reconstitution and in vitro transcription data, since Francisella RNAP containing $\alpha 1$ homodimer is functional and formation of an $(\alpha 1)_{2} \beta$ intermediate can be detected in vitro, albeit with low efficiency. The latter result suggests that $\beta$ may stimulate $\alpha 1$ dimerization. An alternative possibility would be $\beta$ interacting with one $\alpha 1$ monomer, followed by association with another $\alpha 1$ and $\beta^{\prime}$, or the $\alpha 1 \beta^{\prime}$ complex. Be that as it may, our data suggest that bacteria of Francisella genus produce a major form of RNAP containing an oriented $\alpha 1 \alpha 2$ heterodimer, and a minor form containing $\alpha 1$ homodimer.

As shown earlier by hydroxyl-radical-mediated proteolysis [14], the segments of $E$. coli $\alpha$ most strongly protected by $\beta$ correspond to amino acids $30-55$ and $65-75$, and the segments of $\alpha$ most strongly protected by $\beta$ ' correspond to amino acids 175-185 and 195-210. Single alanine substitutions of E. coli $\alpha \mathrm{Lys}^{86}$ and $\mathrm{Val}^{173}$ and two-amino-acid insertions at positions 180 and 200 of $E$. coli $\alpha$ cause defects in $\beta$ ' binding without affecting the $\alpha_{2} \beta$ assembly formation $[16,17]$. To evaluate the ability of Francisella $\alpha 1$ and $\alpha 2$ subunits to interact with the $\beta$ ' subunit, we compared sequences of E. coli $\alpha$ subunit involved in interaction with $\beta^{\prime}[14,21]$ to those in $F$. tularensis $\alpha 1$ and $\alpha 2$ subunits (Additional file 1). The results reveal that a lysine at a position corresponding to $E$. coli $\alpha$ position 86 is present in both $\alpha$ polypeptides from Francisella, while amino acids corresponding to $E$. coli $\alpha \mathrm{Val}^{173}$ are, respectively, a valine and a leucine in $\alpha 1$ and $\alpha 2$. Similarly, the site of one two-amino-acid insertion that destroys $\beta$ ' interaction with $\alpha_{2} \beta$ in $E$. coli, $\mathrm{Val}^{180}$, has as its counterpart a valine in $\alpha 2$ and an isoleucine in $\alpha 1$ of Francisella. These conservative changes are unlikely to affect the efficiency of $\beta$ ' binding by the $\alpha$ polypeptides. Interestingly, the site of the residue at the site of the second insertion affecting $\beta$ ' interaction with $\alpha_{2} \beta$ in E. coli, Lys ${ }^{200}$, is conserved in Francisella $\alpha 2$ and $\alpha$ subunits from other bacteria (see Additional file 1), but is substituted with threonine in $\alpha 1$. The results thus implies that Francisella $\beta$ ' interacts with $\alpha 2$. Further experiments will be needed to prove this conjecture.

Our phylogenetic analysis indicates that none of the Francisella rpoA genes was transferred from outside of the $y$-proteobacterial lineage. In fact, both genes most 
likely emerged through duplication of an ancestral single gene followed by acceleration of evolutionary rate of both paralogs. Acceleration of rpoA evolutionary rate after the duplication apparently was accompanied by subfunctionalization of Francisella $\alpha$ subunits, ultimately leading to accumulation of substitutions in residues responsible for homodimerization and involved in the $(\beta$ and $\beta^{\prime}$ subunit interaction.

Similar events, albeit on much longer time intervals, must have led to formation of two very different $\alpha$-like subunits in eukaryotes and archaea. The large $\alpha$-like subunit (RPB3 in eukaryal RNAP II, AC40 in RNAP I and RNAP III, Rpo3 (also known as RpoD) in archaea) heterodimerizes with its much smaller counterpart (RPB11 in eukaryal RNAP II, AC19 in RNAP I and RNAP III, Rpo11 (also known as RpoL) in archaea) [33-36]. Crystallographic and functional analyses indicates that large $\alpha$ homolog makes interaction with the second-largest ( $\beta$-like) subunit through a surface that contains residue homologous to E. coli $\alpha \operatorname{Arg}^{45}[35,37]$, and is thus formally similar to Francisella $\alpha 1$. The smaller $\alpha$ homolog of eukaryal and archaeal RNAP thus corresponds to Francisella $\alpha 2$. One should not take this analogy to far though, since in eukaryotes and archaea, the $\alpha$ heterodimer is not sufficient for recruitment of large RNAP subunits in the complex. Eukaryal RPB10 and RPB12 and their archaeal homologs Rpo10 (also known as RpoN) and Rpo12 (also known as RpoP) form a stable complex with all four polypeptides playing an essential role in assembly and stability of the RNAP complex [36,38,39].

Evolution of the rpoA duplication presented here is one of the best demonstrations in support of the Lynch's subfunctionalization scenario where both copies are subject to relaxed selection and acceleration of the evolutionary rates but rarely develop a new or specialized function [40]. The fact that bacterial RNAP $\alpha$ subunit functions as a dimer should make it particularly prone to duplication/subfunctionalization. Indeed, while the impetus for our study came from an apparently unique situation with two different $\alpha$ subunits in Francisella, bioinformatics analysis revealed additional instances of rpoA duplications, some fairly recent, like in $S$. avermitilis, others more ancient, like in Chloroflexus species. Despite the fact that these RpoA paralogs are being ancestral for the Chloroflexus species, no drastic substitutions in regions responsible for dimerization and/or $\beta / \beta$ ' interactions have accumulated, suggesting that in contrast to the situation observed in Fransicella, the two $\alpha$ subunits of Chloroflexus may still be functionally equivalent. It is likely that many more instances of rpo $A$ duplications and subfunctionalization will be found in the future.

\section{Conclusions}

The data presented here support the following conclusions: (1) only Francisella $\alpha$-heterodimer $(\alpha 1 \alpha 2)$ can be efficiently assembled in vitro; (2) strong direct interactions between $\alpha 1$ NTD and $\alpha 2$ NTD only have been detected in the bacterial two-hybrid system; (3) $\beta$ interacts more efficiently when both of $\alpha 1$ and $\alpha 2$ presented in the reconstitution mix; (4) interaction between $\alpha 1$ and $\beta$ subunits was observed to be stronger than interaction between $\alpha 2$ and $\beta$; (5) based on phylogenetic analysis, two rpoA copies in Francisella most likely must have emerged through a duplication followed by acceleration of the evolutionary rates of both paralogs.

\section{Methods}

\section{Bacterial strains}

E. coli NovaBlue Singles competent cells (Novagen) were used for initial cloning and plasmid propagation. E. coli BL21 (DE3) cells were used for protein overproduction. Reporter E. coli strain FW102 F'O ${ }_{\mathrm{L}} 2-62$ [41] was used for bacterial two-hybrid experiments.

\section{Cloning and expression}

Francisella tularensis novicida genomic DNA has been provided by Dr. Michael Ibba (Ohio State University). Primers for PCR amplification of rpo genes were designed using Francisella tularensis subsp. novicida (FTN) strain U112 genome sequence data [GenBank: NC_008601]. The primers allowed cloning of amplified FTN rpo genes in pET series $E$. coli expression plasmids between the NcoI and EcoRI (or XhoI to express C-terminally hexahistidine-tagged $\alpha$ subunits) restriction sites. The plasmids pET28-FtnA1His, pET28-FtnA2His, pET28-FtnA1, pET28-FtnA2, and pET28-FtnB, pET30FtnC overexpressing, respectively, C-terminally hexahistidine-tagged $\alpha \mathrm{lHis}_{6}$ and $\alpha 2 \mathrm{His}_{6}$, and untagged $\alpha 1, \alpha 2$, and $\beta$, and $N$-terminally hexahistidine-tagged $\beta$ ' subunits, were constructed using routine cloning methods and verified by sequencing of entire rpo portions for each plasmid. BL21 (DE3) cells harbouring the pET28arpo-gene plasmids were grown in $500 \mathrm{ml}$ of LB medium, supplemented with $25 \mu \mathrm{g} / \mathrm{ml}$ kanamycin, at $37^{\circ} \mathrm{C}$ until an $\mathrm{OD}_{600}$ of around 1 was reached. Then the culture was induced to express an RNAP subunit by the addition of $1 \mathrm{mM}$ isopropyl $\beta$-D-1-thiogalactopyranoside and allowed to grow for 2-4 hours. Cells were harvested by centrifugation and stored at $-80^{\circ} \mathrm{C}$ before use.

\section{Protein purification}

Frozen cells were thawed and lysed by sonication in a buffer containing $40 \mathrm{mM}$ Tris- $\mathrm{HCl}, \mathrm{pH} 8.0,0.1 \mathrm{M} \mathrm{NaCl}$, $10 \mathrm{mM}$ EDTA, $10 \mathrm{mM}$ 2-ME. The lysate was clarified by centrifugation for $30 \mathrm{~min}$ at $15,000 \times \mathrm{g}$, and 
supernatant was supplemented with ammonium sulphate to precipitate soluble proteins $\left(\alpha 2, \alpha 1 \mathrm{His}_{6}\right.$, $\left.\alpha 2 \mathrm{His}_{6}\right)$. Pellets containing insoluble proteins $(\alpha 1, \beta$, $\left.\alpha 1 \mathrm{His}_{6}, \alpha 2 \mathrm{His}_{6}, \mathrm{His}_{6}-\beta^{\prime}\right)$ were resuspended in $40 \mathrm{mM}$ Tris- $\mathrm{HCl}, \mathrm{pH}$ 8.0, $0.3 \mathrm{M} \mathrm{KCl}, 10 \mathrm{mM}$ EDTA, 0.2\% Nadeoxycholate to prepare inclusion bodies as described before $[22,42]$. Ammonium sulphate precipitate was collected by centrifugation, and the pellet, containing untagged protein $(\alpha 2)$, was dissolved in $40 \mathrm{mM}$ Tris$\mathrm{HCl}, \mathrm{pH}$ 8.0, $0.1 \mathrm{M} \mathrm{NaCl}, 1 \mathrm{mM}$ EDTA, $2 \mathrm{mM}$ 2-ME, $0.1 \mathrm{mM}$ PMSF and loaded onto a $1 \mathrm{ml}$ HiTrap Heparin HP column (GE Healthcare) equilibrated in the same buffer. The bound protein was eluted by a linear gradient of $\mathrm{NaCl}$ (from 0.1 to $1.0 \mathrm{M}$ ) in the same buffer. The pellet containing $\alpha 1 \mathrm{His}_{6}$ or $\alpha 2 \mathrm{His}_{6}$ was dissolved in 20 mM Tris- $\mathrm{HCl}, \mathrm{pH} 8.0,0.5 \mathrm{M} \mathrm{NaCl}, 2 \mathrm{mM}$ 2-ME, 0.1 $\mathrm{mM}$ PMSF, $5 \mathrm{mM}$ imidazole and loaded onto the $1 \mathrm{ml}$ HiTrap Chelating HP column (GE Healthcare) charged with $\mathrm{Ni}^{2+}$. The column was washed and bound protein was step-eluted with 20, 50, or $100 \mathrm{mM}$ imidazole. Fractions containing pure protein were pooled and dialyzed against two changes of 500 volumes of $40 \mathrm{mM}$ Tris$\mathrm{HCl}, \mathrm{pH}$ 8.0, $100 \mathrm{mM} \mathrm{NaCl}, 0.5$ mM EDTA, 0.5 mM 2ME, $10 \%$ glycerol, concentrated by ultrafiltration using Microsep (PALL, Life Science) centrifugal device, and stored at $-80^{\circ} \mathrm{C}$.

\section{In vitro protein interaction experiments}

Purified proteins were mixed together in pairwise combinations of untagged and his-tagged proteins. Before mixing, proteins in inclusion bodies were solubilised in denaturing buffer containing $6 \mathrm{M}$ guanidine- $\mathrm{HCl}, 20 \mathrm{mM}$ Tris- $\mathrm{HCl}, \mathrm{pH} 8,10 \mathrm{mM} \mathrm{MgCl} 2,10 \mu \mathrm{M} \mathrm{ZnCl} 2,1 \mathrm{mM}$ EDTA, 10mM DTT, 10\% glycerol. Coupled proteins were mixed in $0.5-1 \mathrm{ml}$ of denaturing buffer at equimolar ratio and adjusted to $0.2-0.5 \mathrm{mg} / \mathrm{ml}$ total protein concentration. Refolding of denatured molecules was achieved by removing guanidine- $\mathrm{HCl}$ from reaction mix through one-change dialysis against $500 \mathrm{ml}$ of reconstitution buffer (20 mM Tris- $\mathrm{HCl}, \mathrm{pH} 8,0.2 \mathrm{M} \mathrm{NaCl}, 10$ $\mathrm{mM} \mathrm{MgCl}_{2}, 10 \mu \mathrm{M} \mathrm{ZnCl}, 0.25 \mathrm{mM}$ EDTA, $0.5 \mathrm{mM}$ DTT, 20\% glycerol). Precipitate formed during dialysis was removed by centrifugation. Then supernatant was diluted 4-fold with start buffer (25 mM HEPES, pH 8.0, $0.5 \mathrm{M} \mathrm{NaCl}, 5 \%$ glycerol, $1 \mathrm{mM}$ imidazole) and loaded on $0.5 \mathrm{ml}$ His-Select Nickel Affinity Gel (Sigma) column equilibrated in the same buffer. The column was washed with start buffer, and proteins were eluted with three steps of start buffer containing 20, 100, $200 \mathrm{mM}$ imidazole. Fractions were analyzed by SDS-PAGE and visualized by Coomassie-staining.

Reconstitution of $\alpha_{2} \beta$, and $\alpha_{2} \beta \beta^{\prime}$ was performed as described above. The $\alpha_{2} \beta$ subassemblies were stepwise fractionated on $\mathrm{Ni}^{2+}$-affinity column with 10,50 , and
$100 \mathrm{mM}$ imidazole; $\alpha_{2} \beta \beta^{\prime}$ RNAP core assembly reactions were fractionated by gel-filtration on a Superose 6 column (GE Healthcare) in the buffer containing $40 \mathrm{mM}$ Tris- $\mathrm{HCl}, \mathrm{pH}$ 8.0, $100 \mathrm{mM} \mathrm{NaCl}, 1 \mathrm{mM}$ EDTA, and 1 $\mathrm{mM}$ 2-ME. Superose 6 fractions were checked for transcription activity on a nucleic acid scaffold. For this purpose, nucleic acid scaffold containing radioactively labelled 8-nt RNA primer (Figure 3A) was added into $10 \mu \mathrm{l}$ of the target Superose 6 fraction to obtain artificial transcription elongation complexes and transcription was initiated by the addition of NTP and $\mathrm{Mg}^{2+}$. Reaction products were resolved by denaturing 20\% PAGE and revealed by autoradiography.

\section{Bacterial two-hybrid assays}

Gene fragments encoding Francisella $\alpha 1$ NTD (residues 1-244), $\alpha 2$ NTD (residues 1-242), $\alpha 1 C T D$ (residues 227323 ), $\alpha 2$ CTD (residues 226-318), were cloned in the plasmids $\mathrm{pBR} \alpha \mathrm{LN}$ and $\mathrm{pAC} \lambda \mathrm{cI}$ to gain fusions with $E$. coli RNAP $\alpha$ NTD and bacteriophage $\lambda$ cI proteins respectively $[24,43,44]$. FW102 F'O $_{\mathrm{L}} 2-62$ reporter strain cells were co-transformed with every possible pairwise plasmid combination. Individual transformants were selected and grown in $2 \mathrm{ml}$ of LB medium supplemented with $50 \mu \mathrm{g} / \mathrm{ml}$ carbenicillin, $25 \mu \mathrm{g} / \mathrm{ml}$ kanamycin, 25 $\mu \mathrm{g} / \mathrm{ml}$ chloramphenicol, and $0.1 \mathrm{mM}$ IPTG. $\beta$-galactosidase assays were performed as described earlier [43].

\section{RpoA comparative analysis}

For comparative analysis of two RpoA paralogs in Francisella we retrieved the RefSeq database (NCBI) containing 1055 completely sequenced bacterial genomes on March 2010. A set of 368 RpoA sequences from 355 representative genomes was aligned with MUSCLE program [45], and the maximum likelihood tree for 246 informative aligned positions was built using FastTree program [46]. RAxML program [31] was used for reconstruction of the phylogenetic tree for $\gamma$-proteobacteria with $\beta$-proteobacteria as an outgroup. The same program was used for comparison of the maximum likelihood values for the best and constrained trees. The evolutionary model for tree reconstitution (WAG [47] with gamma-distributed evolutionary rates) was selected with ProtTest program [48].

\section{Additional material}

Additional file 1: Alpha alignment.

Additional file 2: Constrained trees.

\section{Acknowledgements}

The authors thank Y. Wolf and S. Nechaev for critical reading of the manuscript and useful discussions. The project was supported by NIAID 
Award Al090558 to KK. KSM is supported by the intramural funds of the US Department of Health and Human Services (National Library of Medicine). This work was partially supported by an NIH GM59295 grant and Russian Academy of Sciences Presidium program grant in Molecular and Cellular Biology to KS.

\section{Author details}

${ }^{1}$ Department of Biochemistry and Molecular Biology and Waksman Institute of Microbiology, Rutgers, the State University of New Jersey, Piscataway, NJ 08854, USA. ${ }^{2}$ National Center for Biotechnology Information, National Library of Medicine, National Institutes of Health, 8600 Rockville Pike, Bethesda, Maryland 20894, USA. ${ }^{3}$ Institutes of Gene Biology and Molecular Genetics, Russian Academy of Sciences, Moscow, Russia.

\section{Authors' contributions}

DM, KK, KS designed and interpreted the research; DM, KK performed molecular cloning, protein preparations, binding and transcription reactions and analyzed data obtained; KSM designed, performed and interpreted the bioinformatics study; the manuscript was prepared by KK, KSM, KS; all authors read and approved the final manuscript.

Received: 19 August 2011 Accepted: 22 November 2011

Published: 22 November 2011

\section{References}

1. Charity JC, Costante-Hamm MM, Balon EL, Boyd DH, Rubin EJ, Dove SL: Twin RNA polymerase-associated proteins control virulence gene expression in Francisella tularensis. PLoS Pathog 2007, 3(6):e84.

2. Zakharova N, Hoffman PS, Berg DE, Severinov K: The largest subunits of RNA polymerase from gastric helicobacters are tethered. $J$ Biol Chem 1998, 273(31):19371-19374.

3. Zakharova N, Paster BJ, Wesley I, Dewhirst FE, Berg DE, Severinov KV: Fused and overlapping rpoB and rpoC genes in Helicobacters, Campylobacters, and related bacteria. J Bacterio/ 1999, 181(12):3857-3859.

4. Bergsland KJ, Haselkorn R: Evolutionary relationships among eubacteria, cyanobacteria, and chloroplasts: evidence from the rpoC1 gene of Anabaena sp. strain PCC 7120. J Bacteriol 1991, 173(11):3446-3455.

5. Igarashi K, Fujita N, Ishihama A: Identification of a subunit assembly domain in the alpha subunit of Escherichia coli RNA polymerase. $J \mathrm{Mol}$ Biol 1991, 218(1):1-6.

6. Hayward RS, Igarashi K, Ishihama A: Functional specialization within the alpha-subunit of Escherichia coli RNA polymerase. J Mol Biol 1991, 221(1):23-29.

7. Blatter EE, Ross W, Tang H, Gourse RL, Ebright RH: Domain organization of RNA polymerase alpha subunit: C-terminal 85 amino acids constitute a domain capable of dimerization and DNA binding. Cell 1994, 78(5):889-896

8. Igarashi K, Ishihama A: Bipartite functional map of the E. coli RNA polymerase alpha subunit: involvement of the C-terminal region in transcription activation by CAMP-CRP. Cell 1991, 65(6):1015-1022.

9. Ross W, Gosink KK, Salomon J, Igarashi K, Zou C, Ishihama A, Severinov K, Gourse RL: A third recognition element in bacterial promoters: DNA binding by the alpha subunit of RNA polymerase. Science 1993, 262(5138):1407-1413.

10. Ishihama A: Protein-protein communication within the transcription apparatus. J Bacteriol 1993, 175(9):2483-2489.

11. Zillig W, Palm P, Heil A: Function and Reassembly of Subunits of DNAdependent RNA Polymerase. In RNA Polymerase. Volume 6. Edited by: R Losick \& M Chamberlin. Cold Spring Harbor, NY: Cold Spring Harbor Laboratory Press; 1976:101-125.

12. Ishihama A: Subunit of assembly of Escherichia coli RNA polymerase. Adv Biophys 1981, 14:1-35.

13. Kimura M, Fujita N, Ishihama A: Functional map of the alpha subunit of Escherichia coli RNA polymerase. Deletion analysis of the aminoterminal assembly domain. J Mol Biol 1994, 242(2):107-115.

14. Heyduk T, Heyduk E, Severinov K, Tang H, Ebright RH: Determinants of RNA polymerase alpha subunit for interaction with beta, beta', and sigma subunits: hydroxyl-radical protein footprinting. Proc Natl Acad Sci USA 1996, 93(19):10162-10166.
15. Igarashi K, Fujita N, Ishihama A: Sequence analysis of two temperaturesensitive mutations in the alpha subunit gene (rpoA) of Escherichia coli RNA polymerase. Nucleic Acids Res 1990, 18(20):5945-5948.

16. Kimura M, Ishihama A: Functional map of the alpha subunit of Escherichia coli RNA polymerase: amino acid substitution within the amino-terminal assembly domain. J Mol Biol 1995, 254(3):342-349.

17. Kimura M, Ishihama A: Functional map of the alpha subunit of Escherichia coli RNA polymerase: insertion analysis of the aminoterminal assembly domain. J Mol Biol 1995, 248(4):756-767.

18. Murakami K, Kimura M, Owens JT, Meares CF, Ishihama A: The two alpha subunits of Escherichia coli RNA polymerase are asymmetrically arranged and contact different halves of the DNA upstream element. Proc Natl Acad Sci USA 1997, 94(5):1709-1714.

19. Estrem ST, Ross W, Gaal T, Chen ZW, Niu W, Ebright RH, Gourse RL: Bacterial promoter architecture: subsite structure of UP elements and interactions with the carboxy-terminal domain of the RNA polymerase alpha subunit. Genes Dev 1999, 13(16):2134-2147.

20. Cramer P: Multisubunit RNA polymerases. Curr Opin Struct Biol 2002, 12(1):89-97.

21. Zhang G, Darst SA: Structure of the Escherichia coli RNA polymerase alpha subunit amino-terminal domain. Science 1998, 281(5374):262-266.

22. Borukhov S, Goldfarb A: Recombinant Escherichia coli RNA polymerase: purification of individually overexpressed subunits and in vitro assembly. Protein Expr Purif 1993, 4(6):503-511.

23. Ebright $\mathrm{RH}$, Busby S: The Escherichia coli RNA polymerase alpha subunit: structure and function. Curr Opin Genet Dev 1995, 5(2):197-203.

24. Dove SL, Joung JK, Hochschild A: Activation of prokaryotic transcription through arbitrary protein-protein contacts. Nature 1997 386(6625):627-630

25. Zalenskaya K, Lee J, Gujuluva CN, Shin YK, Slutsky M, Goldfarb A: Recombinant RNA polymerase: inducible overexpression, purification and assembly of Escherichia coli rpo gene products. Gene 1990 89(1):7-12.

26. Tang H, Severinov K, Goldfarb A, Ebright RH: Rapid RNA polymerase genetics: one-day, no-column preparation of reconstituted recombinant Escherichia coli RNA polymerase. Proc Natl Acad Sci USA 1995 92(11):4902-4906.

27. Korzheva N, Mustaev A, Kozlov M, Malhotra A, Nikiforov V, Goldfarb A, Darst SA: A structural model of transcription elongation. Science 2000, 289(5479):619-625

28. Battistuzzi FU, Feijao A, Hedges SB: A genomic timescale of prokaryote evolution: insights into the origin of methanogenesis, phototrophy, and the colonization of land. BMC Evol Biol 2004, 4:44.

29. Ciccarelli FD, Doerks T, von Mering C, Creevey CJ, Snel B, Bork P: Toward automatic reconstruction of a highly resolved tree of life. Science 2006, 311(5765):1283-1287.

30. Park HK, Yoon JW, Shin JW, Kim JY, Kim W: rpoA is a useful gene for identification and classification of Streptococcus pneumoniae from the closely related viridans group streptococci. FEMS Microbiol Lett 2010, 305(1):58-64.

31. Stamatakis A: RAxML-VI-HPC: maximum likelihood-based phylogenetic analyses with thousands of taxa and mixed models. Bioinformatics 2006, 22(21):2688-2690.

32. Kannan N, Chander P, Ghosh P, Vishveshwara S, Chatterji D: Stabilizing interactions in the dimer interface of alpha-subunit in Escherichia coli RNA polymerase: a graph spectral and point mutation study. Protein Sci 2001, 10(1):46-54.

33. Lalo D, Carles C, Sentenac A, Thuriaux P: Interactions between three common subunits of yeast RNA polymerases I and III. Proc Natl Acad SC USA 1993, 90(12):5524-5528.

34. Larkin RM, Guilfoyle TJ: Reconstitution of yeast and Arabidopsis RNA polymerase alpha-like subunit heterodimers. J Biol Chem 1997, 272(19):12824-12830

35. Naryshkina T, Rogulja D, Golub L, Severinov K: Inter- and intrasubunit interactions during the formation of RNA polymerase assembly intermediate. J Biol Chem 2000, 275(40):31183-31190.

36. Werner F, Weinzierl RO: A recombinant RNA polymerase II-like enzyme capable of promoter-specific transcription. Mol Cell 2002, 10(3):635-646.

37. Cramer P, Bushnell DA, Fu J, Gnatt AL, Maier-Davis B, Thompson NE, Burgess RR, Edwards AM, David PR, Kornberg RD: Architecture of RNA 
polymerase II and implications for the transcription mechanism. Science 2000, 288(5466):640-649.

38. Werner F, Grohmann D: Evolution of multisubunit RNA polymerases in the three domains of life. Nat Rev Microbiol 9(2):85-98.

39. Werner F, Eloranta JJ, Weinzierl RO: Archaeal RNA polymerase subunits $F$ and $\mathrm{P}$ are bona fide homologs of eukaryotic RPB4 and RPB12. Nucleic Acids Res 2000, 28(21):4299-4305.

40. Lynch M, Conery JS: The evolutionary demography of duplicate genes. Struct Funct Genomics 2003, 3(1-4):35-44.

41. Deaconescu AM, Chambers AL, Smith AJ, Nickels BE, Hochschild A, Savery NJ, Darst SA: Structural basis for bacterial transcription-coupled DNA repair. Cell 2006, 124(3):507-520.

42. Tang H, Kim Y, Severinov K, Goldfarb A, Ebright RH: Escherichia coli RNA polymerase holoenzyme: rapid reconstitution from recombinant alpha, beta, beta', and sigma subunits. Methods Enzymol 1996, 273:130-134.

43. Dove SL, Hochschild A: A bacterial two-hybrid system based on transcription activation. Methods Mol Biol 2004, 261:231-246.

44. Hu JC, Kornacker MG, Hochschild A: Escherichia coli one- and two-hybrid systems for the analysis and identification of protein-protein interactions. Methods 2000, 20(1):80-94.

45. Edgar RC: MUSCLE: a multiple sequence alignment method with reduced time and space complexity. BMC Bioinformatics 2004, 5:113.

46. Price MN, Dehal PS, Arkin AP: FastTree 2-approximately maximumlikelihood trees for large alignments. PLoS One 2010, 5(3):e9490.

47. Whelan S, Goldman N: A general empirical model of protein evolution derived from multiple protein families using a maximum-likelihood approach. Mol Biol Evol 2001, 18(5):691-699.

48. Darriba D, Taboada GL, Doallo R, Posada D: ProtTest 3: fast selection of best-fit models of protein evolution. Bioinformatics 2011, 27(8):1164-1165.

doi:10.1186/1471-2199-12-50

Cite this article as: Mukhamedyarov et al.: Francisella RNA polymerase contains a heterodimer of non-identical $\alpha$ subunits. BMC Molecular Biology 2011 12:50.

\section{Submit your next manuscript to BioMed Central and take full advantage of:}

- Convenient online submission

- Thorough peer review

- No space constraints or color figure charges

- Immediate publication on acceptance

- Inclusion in PubMed, CAS, Scopus and Google Scholar

- Research which is freely available for redistribution

Submit your manuscript at www.biomedcentral.com/submit 